\title{
EXTENSION OF CFD CODES APPLICATION TO TWO- PHASE FLOW SAFETY PROBLEMS
}

\author{
DOMINIQUE BESTION \\ CEA-Grenoble, DEN-DER-SSTH \\ 17 rue des Martyrs, 38054, GRENOBLE, FRANCE \\ "Corresponding author. E-mail : dominique.bestion@cea.fr
}

Received July 15, 2010

This paper summarizes the results of a Writing Group on the Extension of CFD codes to two-phase flow safety problems, which was created by the Group for Analysis and Management of Accidents of the Nuclear Energy Agency's Committee on the Safety of Nuclear Installations (NEA-CSNI). Two-phase CFD used for safety investigations may predict small scale flow processes, which are not seen by system thermalhydraulic codes. However, the two-phase CFD models are not as mature as those in the single phase CFD and potential users need some guidance for proper application. In this paper, a classification of various modelling approaches is proposed. Then, a general multi-step methodology for using two-phase-CFD is explained, including a preliminary identification of flow processes, a model selection, and a verification and validation process. A list of 26 nuclear reactor safety issues that could benefit from investigations at the CFD scale is identified. Then, a few issues are analyzed in more detail, and a preliminary state-of-the-art is proposed and the remaining gaps in the existing approaches are identified. Finally, guidelines for users are proposed.

KEYWORDS : Two-phase Flow, Thermalhydraulics, CFD, Nuclear Safety

\section{INTRODUCTION}

The OECD/NEA/CSNI has promoted activities with the hope of applying Computational Fluid Dynamics (CFD) to nuclear reactor safety. Three Writing Groups were created under the auspices of the Working Group for the Analysis and Management of Accidents (WGAMA) to produce state-of-the-art reports on different aspects of the subject. The first group, WG1, established Best Practice Guidelines [1] for CFD application to the field of Nuclear Reactor Safety (NRS). The second group, WG2, documented the existing assessment databases [2] for CFD application to some identified NRS issues. The third group, WG3, established some requirements for extending CFD codes to two-phase flow safety problems. The group worked for several years on these projects (2003-2009) and produced two reports [3, 4]. The present paper summarises the content of the reports, with some focus on a few important aspects from the reports.

Extending CFD codes to two-phase flow allows for safety investigations to get some access to smaller scale flow processes that are not seen by system codes. Using such tools as part of a safety demonstration may bring a better understanding of physical situations, which would result in more confidence in one's results, and a better estimation of safety margins. The increasing computer performance allows for a more extensive use of $3 \mathrm{D}$ modelling of two-phase thermalhydraulics with finer nodalization. However, the two-phase flow models are not as mature as those in single phase CFD and a lot of work needs to be done on the physical modelling and numerical schemes in such two-phase CFD tools.

The WG3 first identified and classified the NRS problems, where extending CFD to two-phase flow may bring a real benefit and also classified different modelling approaches. A general multi-step methodology was proposed, including a preliminary identification of flow processes, a model selection, and a verification and validation process. Then, only 6 NRS problems where two-phase CFD may bring real benefits were selected to be analysed in more detail. These problems are the Dryout, the Departure from Nucleate Boiling (DNB), Pressurised Thermal Shock (PTS), pool heat exchanger, steam discharge in a pool, and fire events. These are issues where some investigations are currently ongoing and their CFD investigations have a reasonable chance to be successful in a reasonable period of time. These investigations address all flow regimes so that they may, to some extent, envelop many other issues. The general multi-step methodology was applied to each issue to identify the gaps in the existing approaches. Basic processes were identified. Modelling options were discussed, including closure relations for interfacial transfers, turbulent transfers, and wall transfers. Available data for 
validation were reviewed and the need for additional data was identified. Verification tests were also identified. A few benchmarks were proposed for future activity. A preliminary state-of-the-art was reported that identified the remaining gaps in the existing approaches.

Although the two-phase CFD is still not very mature, the Best Practice Guidelines (BPG) was created which should be complemented and updated in the future. The proposed multi-step methodology allows for a first set of BPG to be implemented for two-phase CFD by inviting users to formulate and justify all their choices and by listing some necessary consistency checks. Some methods for controlling the numerical errors were also given as a part of the BPG.

In this paper, the proposed classification of various modelling approaches is presented in detail. The proposed multi-step methodology for using two-phase CFD is also reported. A list of 26 nuclear reactor safety issues that could benefit from investigations at the CFD scale is given. Then, a few selected issues are analyzed in more detail and a preliminary state-of-the-art is proposed that identifies the remaining gaps in the existing approaches for each issue. Finally, guidelines for users are proposed.

\section{REVIEW OF SAFETY ISSUES THAT MAY BENEFIT FROM TWO-PHASE CFD}

A list of 26 NRS problems where two-phase CFD may bring a real benefit has been established. These issues have been analysed and classified with respect to the degree of maturity of the present tools for solving them in a short or medium term. Then, a limited number of issues became the focus.

Only modelling of two-phase flow configurations pertinent to NRS problems were considered. The NRS problems were classified in terms of maturity of CFD technology applicable. Three categories (Low, Medium, and High) were employed after considering the availability of information, the identification of phenomena, and the existence of models:

- 'High' maturity was applied to the case in which sufficient information was available, all related phenomena were well identified, and models were developed for each phenomenon. Improvements may be welcome for some of them.

- "Medium" maturity was applied when a publicized background exists. Most basic phenomena are supposed to be well identified and some models exist that require improvements and validation.

- 'Low' maturity was applied to the case in which no trusted information was available on the validity of existing models.

Some NRS problems require two-phase CFD in an open medium, while others require a two-phase CFD in a porous medium. For some problems, investigations with a two- phase CFD tool for an open medium were used for a better understanding of flow phenomena and for developing closure relations of a $3 \mathrm{D}$ model for the porous medium. Table 1 presents this classification.

\section{CLASSIFICATION OF CFD MODELLING APPROACHES}

In contrast to the existing thermalhydraulic system codes, such as RELAP-5, CATHARE or TRACE, which have an extensively validated frozen model (usually the two-fluid model), available CFD codes offer many modelling options and a more limited validation. If twophase CFD codes are to be used in a safety context, some requirements need to be applied to the code and to its verification and validation, which take into account this versatility of the options.

In this regard, the first step is to identify the various modeling options and the WG3 proposed a classification based on the following aspects:

- Porous medium or open medium approach

- Phase averaging or field averaging:

- Homogeneous for a two-phase mixture

- Two-fluid model

- Multi-field models

- Filtering turbulent scales and two-phase intermittency scales:

- All turbulent scales are filtered (RANS type models)

- Only some scales are filtered (LES type models)

- All turbulent scales are predicted (DNS type models)

- Treatment of interfaces

- Use of interface tracking/capturing technique

- Use of a pure statistical treatment of interfaces

- Use of an Identification of the Local Interface Structure (ILIS)

- Characterization of the interfaces through interfacial area density or other quantities

\subsection{Open medium approach and porous body approach}

One may distinguish between the open medium and porous medium approaches. Some differences are given:

Porous medium approach:

- equations are space-averaged over a scale larger than the hydraulic diameter

- the porosity appears in the balance equations

- each cell contains walls

- the transfers with the wall have to be modelled on the right hand side of balance equations

- part of the turbulent transfers is modelled as transfers with walls

- turbulent diffusion terms are also needed

Open medium approach:

- equations are time averaged and possibly space- 
Table 1. Identification and Classification of Two-phase NRS Issues that may Benefit from Investigations at the CFD Scale

\begin{tabular}{|c|c|c|c|}
\hline & NRS problem & $\begin{array}{l}\text { Maturity of present } \\
\text { CFD tools }\end{array}$ & $\begin{array}{l}\text { Open medium } \\
\text { Porous medium }\end{array}$ \\
\hline 1 & DNB, dryout and CHF investigations & M & $\mathrm{O} ; \mathrm{O} \Rightarrow \mathrm{P}$ \\
\hline 2 & Subcooled boiling & M & $\mathrm{O} ; \mathrm{O} \Rightarrow \mathrm{P}$ \\
\hline 3 & Two-phase pressurized thermal shock & M & $\mathrm{O}$ \\
\hline 4 & Thermal fatigue in stratified flows & $\mathrm{L}$ & $\mathrm{O}$ \\
\hline 5 & Direct contact condensation: steam discharge in a pool & M & $\mathrm{O}$ \\
\hline 6 & $\begin{array}{l}\text { Pool heat exchangers: thermal stratification and mixing } \\
\text { problems }\end{array}$ & $\mathrm{H}$ & $\mathrm{O} ; \mathrm{P}$ \\
\hline 7 & Corrosion Erosion deposition & $\mathrm{L}$ & $\mathrm{O}$ \\
\hline 8 & Containment thermal-hydraulics & $\mathrm{H}$ & $\mathrm{O}$ \\
\hline 9 & Two-phase flow in valves, safety valves & $\mathrm{L}$ & $\mathrm{O}$ \\
\hline 10 & ECC bypass and downcomer penetration during refill & $\mathrm{L}$ & $\mathrm{O} ; \mathrm{O} \Rightarrow \mathrm{P}$ \\
\hline 11 & Two phase flow features in BWR cores & M & $\mathrm{P} ; \mathrm{O} \Rightarrow \mathrm{P}$ \\
\hline 12 & Atmospheric transport of aerosols outside containment & M & $\mathrm{O}$ \\
\hline 13 & DBA reflooding & M & $\mathrm{P} ; \mathrm{O} \Rightarrow \mathrm{P}$ \\
\hline 14 & Reflooding of a debris bed & $\mathrm{L}$ & $\mathrm{P} ; \mathrm{O} \Rightarrow \mathrm{P}$ \\
\hline 15 & Steam generator tube vibration & $\mathrm{L}$ & $\mathrm{O} \Rightarrow \mathrm{P}$ \\
\hline 16 & Upper plenum injection & $\mathrm{L}$ & $\mathrm{P}$ \\
\hline 17 & Local 3-D effects in singular geometries & $\mathrm{L}$ & $\mathrm{O}$ \\
\hline 18 & $\begin{array}{l}\text { Phase distribution in inlet and outlet headers of steam } \\
\text { generators }\end{array}$ & $\mathrm{L}$ & $\mathrm{O} ; \mathrm{O} \Rightarrow \mathrm{P}$ \\
\hline 19 & Condensation induced waterhammer & $\mathrm{L}$ & $\mathrm{O}$ \\
\hline 20 & Components with complex geometry & $\mathrm{L}$ & $\mathrm{O} \Rightarrow \mathrm{P}$ \\
\hline 21 & Pipe Flow with Cavitation & M & $\mathrm{O}$ \\
\hline 22 & External reactor pressure vessel cooling & M & $\mathrm{O}$ \\
\hline 23 & Behaviour of gas-liquid interfaces & M & $\mathrm{O}$ \\
\hline 24 & Two-phase pump behaviour & $\mathrm{L}$ & $\mathrm{O}$ \\
\hline 25 & Pipe Break-In vessel mechanical load & M & $\mathrm{O} ; \mathrm{P}$ \\
\hline 26 & Specific features in Passive reactors & M & $\mathrm{O}$ \\
\hline
\end{tabular}

averaged over a scale much smaller than the hydraulic diameter

- no porosity appears in the equations

- cell size is much smaller than the hydraulic diameter

- cells do not contain walls: walls only appear at boundaries of the calculation domain

- the transfers with walls are modelled by wall functions

- turbulent diffusion terms have to be modelled

\subsection{The number of fluids or fields}

One can separate any two-phase flow into one or several fields:
- 1-fluid for a model that considers a mixture of the two phases together

- 2-fluid for a model that considers each phase separately

- n-field for a model that splits one or both phases into several fields.

Multi-fields model are commonly of the type $2+\mathrm{n}_{\mathrm{b}}+$ $\mathrm{n}_{\mathrm{d}}$ fields with two continuous fields (continuous gas and continuous liquid) + one or $n_{b}$ bubble fields +1 or $n_{d}$ droplet fields.

\subsection{Time averaging and space averaging}

Time or ensemble averaging is a common way to derive 
equations for the so-called Reynolds Average NavierStokes (RANS) approach. Although time averaging and ensemble averaging are different, they can be reasonably considered as equivalent (ergodicity) in steady or quasisteady flows where the RANS approach is applied.

Time averaging filters all turbulent scales and predicts only a mean velocity field. Time averaging does not allow for the prediction of space and time position of the interfaces of dispersed droplets and dispersed bubbles. It also has a smearing or diffusive effect on the large interfaces between continuous liquid and continuous gas, such as a free surface or the surface of a liquid film along a wall.

Space averaging or filtering is also used in the socalled Large Eddy Simulation (LES) of turbulent flows in an open medium context. This technique has become increasingly applied in single phase CFD in order to simulate some transient flow or predict large scale coherent structures. The filter scale defines the part of the turbulence spectrum, which is simulated, and the part that must be modelled. Space averaging in two-phase flow filters not only the small eddies, but also the interfaces.

\subsection{Simulated interface, filtered interface, or statistical interface}

A simulated interface is deterministically treated since its space and time position is simulated. In DNS or pseudoDNS techniques, neither space nor time averaging is used.

An interface is statistically treated when its space and time position gets lost in an averaging or filtering procedure. Only statistical or averaged information on several interfaces may be predicted through quantities, such as void fraction or an interfacial area density. Statistical treatment may result from time averaging or from space averaging.

An interface is a filtered interface where its space position and time evolution is predicted with some filtering due to either a space filter or from time averaging.

Based on the classification, one can summarize the various time and space resolution options that are possible in two-phase CFD, as shown in Table 2.

Space filtering affects both the turbulence and the interface simulation or modelling. Three different sub-

Table 2. Time and Space Resolution in the Various Modelling Approaches of Two-phase CFD

\begin{tabular}{|c|c|c|c|c|c|c|}
\hline \multicolumn{7}{|c|}{ TIME AND SPACE RESOLUTION IN TWO-PHASE CFD } \\
\hline \multirow[b]{2}{*}{$\begin{array}{l}\text { Time \& space } \\
\text { filtering }\end{array}$} & \multicolumn{5}{|c|}{ Open medium } & \multirow{2}{*}{\begin{tabular}{|c|} 
Porous medium \\
Time \\
averaging
\end{tabular}} \\
\hline & $\begin{array}{c}\text { No filter } \\
\text { No averaging }\end{array}$ & & Space filtering & & $\begin{array}{c}\text { Time } \\
\text { averaging }\end{array}$ & \\
\hline $\begin{array}{c}\text { Turbulence } \\
\text { DNS } \\
\text { LES } \\
\text { VLES } \\
\text { RANS }\end{array}$ & DNS & LES & $\begin{array}{c}\text { LES } \\
\text { VLES }\end{array}$ & $\begin{array}{c}\text { LES } \\
\text { VLES }\end{array}$ & $\begin{array}{c}\text { RANS } \\
\text { URANS }\end{array}$ & $\begin{array}{c}\text { RANS } \\
\text { URANS }\end{array}$ \\
\hline $\begin{array}{l}\text { Interfaces } \\
\text { Simulated } \\
\text { Filtered } \\
\text { Statistical }\end{array}$ & $\begin{array}{l}\text { Simulated } \\
\text { Interfaces }\end{array}$ & $\begin{array}{l}\text { Simulated } \\
\text { Interfaces }\end{array}$ & $\begin{array}{l}\text { Filtered \& } \\
\text { Statistical } \\
\text { Interfaces }\end{array}$ & $\begin{array}{l}\text { Statistical } \\
\text { Interfaces }\end{array}$ & $\begin{array}{l}\text { Statistical } \\
\text { Interfaces }\end{array}$ & $\begin{array}{l}\text { Statistical } \\
\text { Interfaces }\end{array}$ \\
\hline $\begin{array}{c}\mathrm{Nb} \text { of fields } \\
1-\mathrm{F} \\
2-\mathrm{F} \\
\mathrm{n}-\mathrm{F}\end{array}$ & $1-\mathrm{F}$ & $1-\mathrm{F}$ & $\begin{array}{l}1-\mathrm{F} \\
2-\mathrm{F} \\
\mathrm{n}-\mathrm{F}\end{array}$ & $\begin{array}{l}1-\mathrm{F} \\
2-\mathrm{F} \\
\mathrm{n}-\mathrm{F}\end{array}$ & $\begin{array}{l}1-\mathrm{F} \\
2-\mathrm{F} \\
\mathrm{n}-\mathrm{F}\end{array}$ & $\begin{array}{l}1-\mathrm{F} \\
2-\mathrm{F} \\
n-\mathrm{F}\end{array}$ \\
\hline Type of models & $\begin{array}{l}\text { Pseudo } \\
\text { DNS }\end{array}$ & $\begin{array}{l}\text { LES with } \\
\text { simulated } \\
\text { interfaces }\end{array}$ & $\begin{array}{l}\text { Hybrid LES } \\
\text { with filtered } \\
\text { \& statistical } \\
\text { interfaces }\end{array}$ & $\begin{array}{l}\text { LES with } \\
\text { statistical } \\
\text { interfaces }\end{array}$ & $\begin{array}{c}\text { RANS } \\
\text { URANS } \\
\text { statistical } \\
\text { interfaces }\end{array}$ & $\begin{array}{c}\text { Porous medium } \\
\text { approach } \\
\text { statistical } \\
\text { interfaces }\end{array}$ \\
\hline
\end{tabular}


cases exist in the case of space filtering:

- The filter scale is much smaller than the scales of the interfaces (bubble size, droplet size, wave length at a free surface) and all interfaces are simulated (third column from left in Table 2)

- The filter scale is larger than the scales of the interfaces (e.g. bubble size or droplet size) and no interfaces are simulated (fifth column from left in Table 2). All interfaces are statistically treated

- The filter scale is larger than some interface scales (e.g. small bubble size or droplet size) and smaller than other interface scales (free surface, film interface, interfaces of large bubbles); no interface is simulated; some interfaces are filtered and some interfaces are statistically treated (fourth column from left in Table 2).

\section{MULTI-STEP METHODOLOGY FOR USING CFD FOR NUCLEAR REACTOR SAFETY}

\subsection{The methodology}

The general method illustrated in Figure 1 was proposed for using two-phase CFD for safety issues with these steps below:

1. Identifying all important flow processes

2. Main modelling choices

2.1 Selecting a basic model

2.2 Filtering turbulent scales and two-phase intermittency scales

2.3 Treatment of interfaces

3. Selecting closure laws

3.1 Modelling interfacial transfers

3.2 Modelling turbulent transfers

3.3 Modelling wall transfers

4. Verification

5. Validation

If the CFD tool is used in the context of a nuclear

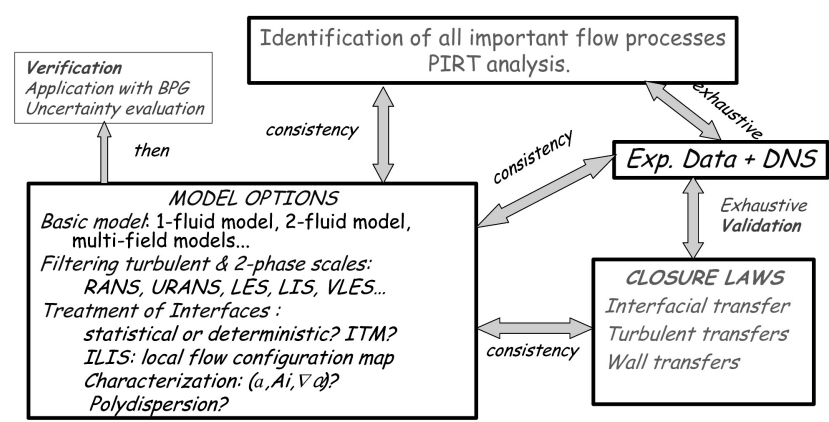

Fig. 1. General Methodology for Two-phase CFD Application to Nuclear Reactor Safety reactor safety demonstration that uses a best-estimate approach, one may add a final step:

6. Uncertainty evaluation

\subsection{Identification of all important flow processes}

Most issues with reactors involve complex two-phase phenomena in a complex geometry with many basic flow processes that may play a role. The user must identify all these basic thermalhydraulic phenomena before selecting various code options that are available in most two-phase CFD codes. None of the available codes can be used as a black box, which could take a complex problem and automatically select the options that can provide the adequate answer. These basic phenomena have to be ranked with respect to the importance of the reactor issue. This can be achieved by performing a Process Identification and Ranking Table (PIRT) analysis or by a similar approach. The preliminary analysis of some experiments simulating the problem (or part of the problem) may be of great help in identifying the phenomena. Considering the inherent complexity of any two-phase flow, this identification of important processes should be revisited several times during the successive steps of the general methodology. Modelling work and validation work may change our mind on the relative importance of each phenomenon. Also, analyzing some experimental data from the validation matrix may highlight some sensitive phenomenon that was not previously identified. The methodology may then be iterative.

\subsection{Main Modelling Choices}

Three choices are necessary to select the set of balance equations that will be used to solve the problem and they must be consistent with each other. These choices are related to separation into fields, time and space filtering, and treatment of interfaces (Section 3).

Any two-phase flow may be seen as a juxtaposition of several fields and/or phases. The separation into several fields is particularly necessary when each field has a velocity and/or a temperature significantly different from the others. In some cases, it may be necessary to separate droplets (bubbles) into several classes that have different sizes, especially when the behaviour depends on the size.

The second important choice is the choice of the type of time and space averaging or filtering of equations. Pseudo-DNS techniques are still too CPU time consuming for real safety application and can only be used as a support for modelling at more macroscopic scales. Filtered approaches are also CPU time consuming, but may already be applied to some investigations. RANS type models are more affordable and are still the privileged way for complex applications.

Depending on the averaging, the interfaces are either simulated (or deterministic), filtered, or statistically treated. Two-phase flows have interfaces with a wide 
range of geometrical configurations. There are locally "closed interfaces" for dispersed fields, e.g. bubbles and drops, and locally open interfaces for free surfaces, interface of a falling film, or of a jet. Simulated or filtered interfaces are more adaptable to "large interfaces", like free surfaces or film surfaces. A pure statistical treatment is more adaptable to disperse flows, such as bubbly flow or droplet flow.

In a RANS context, one may need an "Identification of the Local Interface Structure" (ILIS) to select appropriate closure laws for the interfacial transfers. Such an ILIS is equivalent to the "flow regime map" used in 1D two-fluid models in system codes.

A local interfacial structure is defined by three items:

1. Presence of dispersed gas field (bubbles)

2. Presence of dispersed liquid field (drops)

3. Presence and direction of a "large Interface"

In some cases, one may combine a deterministic treatment of "large interfaces" with a statistical description of dispersed fields.

In a statistical description of interfaces, the interfaces are characterized at least by volume fraction, but very often, additional information provided by additional equations is required for particle number density, interfacial area density, multi-group volume fractions (e.g. MUSIG method), or any other information on the particle population.

\subsection{Selecting Closure Laws}

Any kind of interface may be subject to mass, momentum, and energy interfacial transfer. The formulation of these transfers depends on the modelling choices mentioned above, such as filter scale and interface treatment. If a "Large Interface" (such as a free surface) is present, an adequate model may require knowledge of the precise position of this interface either by using an Interface Tracking Method (ITM) or any other method. When an ILIS has defined the interface structure, the choice of adapted closure laws is possible. All mass momentum and energy interfacial transfers have to be modelled and validated on available Separate Effect Tests (SETs).

Turbulent transfers have to be modelled and validated on available SETs. The formulation of these transfers depends on the above modelling choices on the basic model, filter scale, and interface treatment. On the other hand, a wall-like treatment of large interfaces requires a damping of turbulence in the vicinity of the interface.

Momentum and energy wall transfers have to be modelled (though adequate wall functions) and validated on available SETs.

\subsection{Validation and Verification}

Pure numerical benchmarks may be necessary to check the capabilities of the numerical scheme and to measure the accuracy of the resolution.

A matrix of validation tests (and possibly also of demonstration tests) has to be defined and used. Demonstration tests may be necessary to demonstrate the capability of the modelling approach to capture all basic flow processes, at least qualitatively. Validation tests are necessary to evaluate the models for interfacial, turbulent, and wall transfer terms of the equations, as far as possible by using separate effects.

\section{ANALYSIS OF SOME SELECTED NRS ISSUES}

Six NRS problems where two-phase CFD may bring real benefits were selected to be analysed in more detail. These problems are the Dry-out, the Departure from Nucleate Boiling (DNB), Pressurised Thermal Shock (PTS), pool heat exchanger, steam discharge in a pool, and fire events. Looking at all possible local interface configurations of two-phase flows, there are a limited number of possibilities:

- Dispersed bubbles in a continuous liquid

- Dispersed droplets or particles in a continuous gas phase

- Separate-phase flow at the vicinity of the interface, such as a free surface in a stratified flow, or the surface of a liquid film. In both cases, there are only two continuous fields.

- Presence of two continuous fields and two dispersed fields. This is the case of a stratified flow when droplets are above the liquid surface and bubbles are below the surface. This kind of situation may be found when there are waves with droplets entrained at the wave crests and when breaking waves are trapping bubbles below the free surface.

Looking at all possible heat transfers, there may be heating walls, cooling walls, vaporisation or condensation, and heat sources due to chemical reactions.

Bubbly flows are encountered in DNB investigations and in pool heat exchangers. Droplet flows and particle flows are encountered in dry-out investigations and fire analyses. Free surfaces are encountered in PTS and pool heat exchangers while liquid films are encountered in dry-out investigations. Heating walls and vaporisation are present in both DNB and dry-out investigations, while condensation takes place in PTS and steam discharge in a pool. A free surface with bubbles below the surface is found in pool heat exchangers and at ECC injections during the investigation of PTS. The most complex situation with two continuous fields and two dispersed fields was not present in the selected issues. Considering the relative low maturity of two-phase CFD tools, it is expected that the selected issues gave an opportunity to cover many flow configurations, leaving aside only the most complex situations. The following sub-sections present, in short, the analysis of four of these issues. 


\subsection{Dry-Out investigations}

One of the major limiting factors in the safe operation of Boiling Water Reactors (BWR) is the occurrence of dry out, which manifests itself with the break-up or disappearance of the liquid film in heated annular twophase flows. Due to dry-out, the heat transfer between cladding and coolant significantly deteriorates and, as a result, the cladding temperature rapidly increases. This, in turn, can cause damage to the cladding and can lead to a release of fission products to the coolant. The liquid phase exists as a liquid film, which is attached to walls, and as droplets, which are carried in the central part of the channel by the vapour phase. The mass flow rate in the liquid film is changing due to vaporization, droplet deposition, or entrainment into the vapour core.

The expected major benefits of employing the CFD approach are the capability to capture the geometrical influence on dry-out, including the influence of spacer grids on the drop size and deposition rates, as well as the modelling of turbulence effects on the spatial distribution of drops, distribution of drop sizes, and deposition of drops on liquid film, allowing for more accurate predictions.

The most promising approach which can be applied for annular-mist flows is based on the three-field RANS modelling (see [6]), with a vapour field, liquid film, and dispersed droplet field. The equations are closed with droplet-vapour interaction terms that describe the exchange of mass, momentum, and energy. Another approach could employ Large Eddy Simulation (LES), in which only the smallest eddies, which are smaller than the droplets and wave structure on the liquid film, will be filtered. This approach would enable the inclusion of the wave motion on the film surface, and in particular, would take into account the influence of disturbance waves on the dry-out occurrence. The drop size can be calculated from an algebraic expression as a function of local parameters. In a more sophisticated approach, the drop size can be determined from predicting the drop break-up and collisions by using a transport of the interfacial area equation.

The modelling of the droplet deposition is of major importance. It depends on the drop dispersion in a turbulent flow where particle motion is primarily governed by interactions with eddies of various scales. Depending on the ratio of the particle response time to the eddy characteristic time, the particles can follow the eddy, be dispersed, or be unaffected by eddies. For example, the Lagrangian Particle Tracking technique was used to investigate droplet deposition by Caraghiaur et al. [7, 8].

A data basis about the annular-mist flow and dry-out was collected in the frame of the NURESIM project [9]. Early experiments focused on measuring the total power, which was necessary for the occurrence of dry-out in a heated channel. A vast number of these experiments were performed for different conduit geometries in different flow conditions. The measurements for steam-water were done in round ducts, annuli, and rod clusters. The data were reported in [10].

\subsection{DNB investigations}

The Critical Heat Flux (CHF) in PWR is currently estimated by empirical DNB correlations that have been developed from specific measurements. Extrapolation of correlations beyond the experimental range, in general, is not possible. Any new fuel assembly design requires fullscale measurements. Because of that, the design process is quite costly and time consuming. A better understanding of local flow processes is necessary in the future in order to allow the present industrial methods to be replaced by a "Local Predictive Approach" [11], where CHF correlations will be based on local parameters predicted by CFD, instead of averaged parameters. Even if it is a rather long term objective, such investigations may bring a better understanding and modelling of non-uniform heat flux impact, grid impact, and channel shape/size impact.

The available experimental data base for DNB investigations was collected [9], including adiabatic airwater or steam-water bubbly flow experiments (DEDALE, TOPFLOW), boiling flow data in simple geometry (tubes and annuli), data in more complex geometry (rod bundle data), and boiling flow data in fuel bundle tests.

As boiling bubbly flows are encountered, the two-fluid model is naturally used in these flow conditions, which benefit from the possibility of modelling all interfacial forces acting on the bubbles, such as drag, lift, turbulent dispersion, virtual mass, and wall forces, which control the void repartition in a boiling channel. The choice of method to model poly-dispersion effects remains partly open.

Considering flow in a PWR core in conditions close to nominal, when boiling occurs, a high velocity steady flow regime takes place with times scales associated to the passage of bubbles being very small $\left(10^{-4}, 10^{-3} \mathrm{~s}\right)$ and with the bubble diameter being rather small $\left(10^{-5}\right.$ to $10^{-3}$ $\mathrm{m}$ ) compared to the hydraulic diameter (about $10^{-2} \mathrm{~m}$ ). These are perfect conditions to use a time average or ensemble average of equations, which is usually done in the RANS approach. All turbulent fluctuations and twophase intermittency scales can be filtered since they are significantly smaller than the scales of the mean flow. The use of a Large Eddy Simulation (LES) approach may allow the simulating of bubble dispersion by liquid turbulence instead of modelling it.

Liquid turbulence plays a very important role in boiling flows, influencing the liquid temperature diffusion, bubble dispersion, bubble detachment, bubble coalescence, and break up, which affect the Interfacial area. The k- $\varepsilon$ or Shear Stress Transport (SST) methods were used with some success by Morel et al. [12, 13, 14, 15], Mimouni et al. [16], and Lucas et al, [17]. If the swirling flow past a spacer grid vane must be modelled, it is shown [18] that the SST models can perform better than the k- $\varepsilon$ model. The LES approach has been evaluated in the 
simulations of a bubble column [19]. LES can only be used in situations for which the bubble size is small enough compared to large turbulent eddies, which is not necessarily the case in convective boiling.

Some improvements of wall function for momentum were obtained and validated on ASU tests [20, 21]. More generally, specific wall functions have to be developed for the boiling flow for both momentum and energy equations. Such wall functions should be able to provide a converged solution with a reasonably coarse nodalization close to a heating wall.

With DNB occurrence, a gas layer appears and a criterion must be implemented to identify this occurrence. A very simple criterion based on the local void fraction was applied to LWL tests. However, the description of the interface structure may require additional transport equations, such as Interfacial Area Transport (IAT) or bubble number density transport. More generally, the method of the statistical moments [22] can be used to characterise the poly-dispersion of the vapour phase with a bubble size spectrum. Another approach to polydispersion is to use a Multi-group model (MUSIG method)

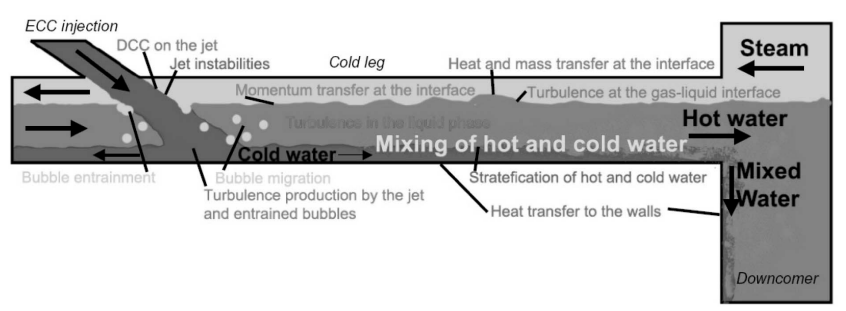

Fig. 2. Most Important flow Phenomena During a PTS Situation with Partially Filled Cold Leg

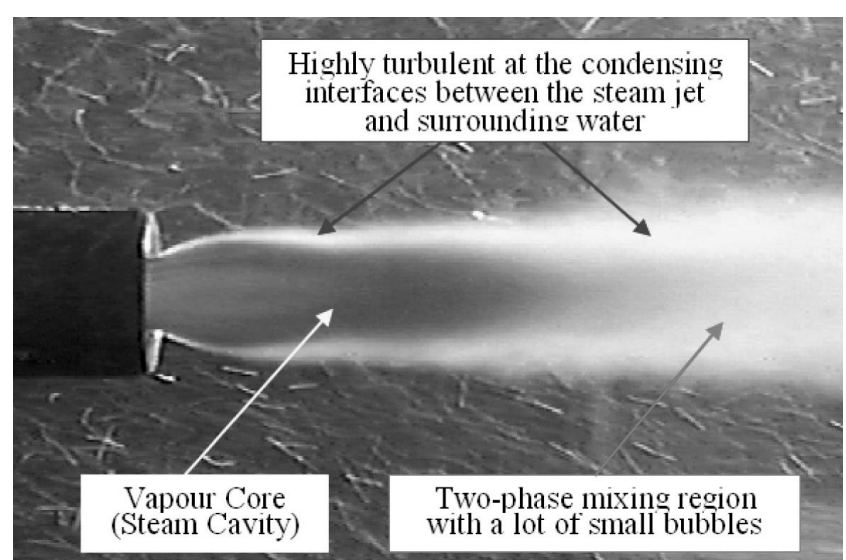

Fig. 3. Steam Jet of High Mass Flux in a Subcooled Pool by Song et al.[37] (Nozzle Dia.: $10.15 \mathrm{~mm}$, Mass Flux: 600 $\mathrm{kg} / \mathrm{m}^{2} \mathrm{~s}$, Pool Temp.: $40^{\circ} \mathrm{C}$ ) with mass (and momentum) equations written for several bubble sizes. These two methods were used [23, 24], evaluated, and compared to the experimental data. The MUSIG method with several mass equations for different bubble sizes, and at least two momentum equations, have shown good capabilities for capturing all qualitative effects in TOPFLOW vertical pipe tests.

First demonstration test cases were performed with CFD calculations of Critical Heat Flux tests [25] in the LWL loop, which is the prototype of WWER core assemblies. Although the simulations were not fully successful, CFD showed interesting capabilities, even with its complex industrial geometry and flow conditions.

\subsection{Steam discharge in a pool with Direct Contact Condensation}

Some reactor systems use a steam discharge in a coldwater pool. An example of this was found in a BWR suppression pool. Also, some new reactor designs have passive safety systems that use a similar principle [36]. Direct contact condensation takes place and some instability with pressure fluctuations may occur. The design of steam injectors may have an important effect on the efficiency of the condensation, as well as on the stability and thermal mixing in the pool. The liquid has a low velocity or is quasi-stagnant, whereas steam may flow into a pool as a jet with very high speed. This leads to a rapid condensation of steam and the resultant turbulent mixing. Usually pressure also oscillates locally when a certain volume of steam is condensed radically, leading to an unstable flow behaviour or thermally non-uniform state in a pool. Song et al. [37] experimentally investigated the shapes of steam jet, the length of the steam jet, heat transfer coefficients, and axial and radial temperature distributions in a steam jet over a wide range of test conditions with different nozzle diameters. The important local interfacial structure to be modelled for the steam discharge in a pool is the shape and dimensions of the vapour core, as shown in Figure 3. At the interface between the vapour core and its surrounding liquid, an interfacial model applicable to the case of separated flow might be used, whereas in the two-phase mixture region, another model, which is applicable to the case of a finely dispersed bubble flow, could be valid.

The RANS approach may be a suitable first choice for the CFD analysis of the steam discharge in a pool. In the continuous liquid phase, the Large Eddy Simulation (LES) approach can be applied [38]. This requires a filter scale smaller than the large eddies of the liquid flow and larger than the bubble size. Compared to the RANS approach, using the LES will allow the simulation of bubble dispersion by the liquid turbulence, instead of modelling it.

Data are needed to validate the CFD tools against both local information [39] on the interfacial area and the 
transfer of momentum, heat, and mass in and around the steam jet, as well as the global information on the temperature and velocity distributions and flow circulation pattern in a pool. A data basis was reported [4], including both basic tests and integral tests.

The first CFD simulations of a turbulent jet were reported by Kang et al. [40, 41]. Although some discrepancies are still under analysis, they prove that such tools, after some modelling and validation effort, will bring some benefits for design studies in the future.

\subsection{Pressurised Thermal Shock in Two Phase situations}

Pressurized Thermal Shock (PTS) may occur in a PWR during the overcooling of the pressure vessel wall in cases where irradiation induced loss of ductility may lead to failure. Small Break Loss of Coolant Accident (LOCA) scenarios exist with an Emergency Core Cooling System (ECCS) injection in a partially or totally uncovered cold leg, where the main heat source of the liquid is due to steam condensation in the cold leg and in the top of the downcomer. Condensation is mainly dependent on the interfacial structure and on the turbulent mixing in the liquid phase. A rather simple interfacial structure of a stratified flow takes place in the cold leg. Also, the rapid progress of computer power allows for the use of fine resolution CFD tools for PTS investigations by using 3D nodalization of the cold legs and of the downcomer. This allows for the prediction of the liquid temperature field, which depends mainly on interfacial heat, and mass transfer related to direct contact condensation of steam on a sub-cooled liquid and on the turbulence diffusion within the liquid.

Many previous studies have supported the belief that turbulence behavior near the interface plays a dominant role in interfacial transfers. For ECC injection cases, the turbulence mainly comes from the impact of the water jet and shear at the wall and at the gas-liquid interface. Thus, as a first step to simulate such scenarios, separate effects in simple geometry have to be investigated, i.e., interfacial friction and turbulence production, interfacial heat transfer, turbulence in a water pool induced by a water jet, in order to establish and validate the developed models.

The identification of all basic flow processes was made in the NURESIM [26] project (see Figure 2) and many phenomena were identified.

In the ECCS jet area:

- Instabilities of the jet from ECC injection,

- Condensation on the jet itself before mixing

- Entrainment and migration of steam bubbles below the water level

- Turbulence production below the jet

In the stratified flow in cold leg:

- Interfacial transfer of momentum at free surface
- Interfacial transfer of heat \& mass at free surface

- Turbulence production in wall shear \& in interfacial shear layers

- Heat transfers with cold leg and RPV walls

- Effects of turbulent diffusion upon condensation

- Interactions between interfacial waves, interfacial turbulence production, and condensation

- Effects of temperature stratification upon turbulent diffusion

- Influence of non-condensable gases on condensation

In the downcomer:

- Flow separation or not in dowcomer at cold leg nozzle Heat transfers with the walls

A rather simple interface structure is encountered in the cold leg of the reactor with a stratified flow having a flat or wavy interface. Only the entrainment of bubbles below the free surface by the ECCS jet may give a somewhat complex interface structure, but it is limited to a small region of the flow. The choice of how to treat the free surface, by using a ITM or not, remains partly open and the NURESIM project started evaluating several options [27] using CFX, FLUENT and NEPTUNE codes both with or without ITM, with surface tension or without, with some algorithm for interface sharpening or without.

The flows in the PTS scenarios are quasi-steady flows. Filtering all turbulent scales should then be appropriate, but it may affect the interfacial wave pattern. If interfacial waves play a role in the process, the way to simulate them or model them in a CFD approach is not clear.

The turbulent diffusion within the liquid phase controls the condensation efficiency and a high turbulent mixing due to the ECCS jet impingement is the main source of turbulence. The $\mathrm{k}-\varepsilon$ model seems to be a reasonable first approach for this situation, but a sufficiently fine meshing is required for the turbulence to be correctly described. Interfacial transfers of heat and momentum (friction force) on the free surface require a specific modelling that take into account the space filter scale imposed by the meshing: the transfer coefficients may depend on the distance to the interface in the same way as the distance to the wall is used in wall functions. However, modelling turbulence and heat transfers at the free surface, in the case of a high interfacial shear and presence of waves with RANS turbulence modelling, requires an additional model.

Yao et al [28] and Coste et al. [29] developed a modelling of free surface flow using a two-fluid approach and a $\mathrm{k}-\varepsilon$ model in each phase. The interface position was precisely determined using the void fraction. Momentum and heat transfers in meshes containing the free surface were treated with an extrapolation of the wall function approach. Such a modelling was found to be rather satisfactory, at least for the momentum interfacial transfers and for turbulence prediction. Further efforts are still needed to improve heat and mass transfers.

Lakehal et al $[30,31]$ have used LES and an ITM to 
investigate stratified counter-current air-water flow with high interfacial shear and developed a specific subgrid scale modelling. Such finer scale simulations may be of great interest to understand the complex interactions at the free surface between friction forces, surface tension, wave propagation, condensation or vaporization, turbulence of both the gas flow and the liquid flow for evaluating more macroscopic modelling, such as RANS approaches, and can be used as complementary to experiments that develop closure relations. This illustrates the benefit of having a multi-scale approach to two-phase CFD.

Several experimental data sources were identified [26] and used for the development, and a partial validation, of physical models. Free surface flows without mass transfer were investigated in three experiments ranging from smooth interface up to wavy interface and KelvinHelmholtz instability [32]. Two experiments have provided information on plunging jets with entrainment of air bubbles and production of turbulence below the free surface. The turbulence induced by a plunging jet was found to be the main source of turbulence in the cold leg and it dramatically influenced the condensation. It was found that the $k-\varepsilon$ turbulence model was not very accurate, but could predict the turbulence intensity reasonably well [33]. Condensation at the free surface of a stratified steamwater flow in a rectangular channel was investigated in two experiments [34, 29] and condensation driven KelvinHelmholtz instability in a horizontal pipe was also investigated in the PMK test facility [35]. Finally, the COSI tests are combined effect tests with several phenomena representative of the PTS scenarios, and a UPTF-TRAM test could simulate at a reactor scale many phenomena, but without condensation.

\section{GUIDELINES FOR USING TWO-PHASE CFD}

A general multi-step method of work for using twophase CFD for safety issues is recommended, as explained in Section 2. Following these steps, and being able to justify what is being done at each step, is a good way to demonstrate that the users actually control the whole process and do not simply rely on simulation tools, which are still relatively immature.

The first step just states that the user should not expect that the CFD code will tell him which flow processes will take place in the problem that needs to be solved. The user must identify these flow processes and then check that the simulation tool is able to describe them, either as it is or after some additional developments are made.

The second and third step will exist as long as precise guidelines lack options for selecting the main model and closure relations. The user must elaborate the rationale for these choices for each application. Feedback from many users and many applications will allow more guidance to users for this step in the future.
In particular, the averaging procedure must be specified to give a clear definition of the principal variables and of the closure terms in the equations. The filtering of the turbulent scales and of two-phase intermittency must be fully consistent.

The last three steps are common to all simulation tools, but further efforts are required to develop adequate experimental, numerical and mathematical tools.

In the application of this multi-step methodology, a few consistency checks are necessary.

1. In the first step, the basic choice of the number of fields must be adapted to the physical situation or to an acceptable degree of simplification of the situation. In particular, if two fields are mechanically and/or thermally uncoupled, and have very different behaviour, they must be treated separately.

2 . In the second step, the averaging procedure must be specified to give a clear definition of the principal variables and of the closure terms in the equations. The filtering of the turbulent scales and of two-phase intermittency must be fully consistent.

3. In the second step, an Interface Tracking Method can only be used if all phenomena having an influence on the interface are also deterministically treated.

4. In the third step, the choice of an adequate interfacial transfer formulation must be consistent with the selected interface treatment, and with the Identification of the Local Interfacial Structure (ILIS).

5. In the fifth step, the SET validation matrix should be exhaustive with respect to all flow processes, which were identified in Step 1.

6. In the fifth step, the SET validation matrix should be able to validate all the interfacial turbulent and wall transfers that were mentioned in Step 1 as playing an important role

7. In the fifth step, the number of measured flow parameters in the validation experiments should be consistent with the complexity of the selected model to validate. A model defined by a set of $n$ equations having a set of $n$ principal variables $X_{i}(i=1, n)$ can be said to be clearly "validable" when one can measure $\mathrm{n}$ parameters giving the $\mathrm{n}$ principal variables.

8. In the fifth step, the averaging of measured variables must be consistent with the averaging of the equations. The proposed multi-step methodology and classification of modelling approaches have given a first approach to Best Practice Guidelines for two-phase CFD by inviting users to formulate and justify all their choices and by listing some necessary consistency checks. The work performed by the Writing Group confirms that two-phase CFD is becoming a useful tool that is complementary to system codes for safety investigations. An estimation of safety margins for any of the selected issues has not been provided yet, but the work has given access to small scale flow processes, which provides a better understanding of physical situations. CFD is already a useful tool for safety 
analysis and may become a tool for safety demonstration when all the steps of the methodology have been correctly addressed, including uncertainty evaluation.

\section{ACKNOWLEDGEMENTS}

The author is grateful to the members of the WG3 group for their valuable contributions to the OECD-NEA reports $[3,4]$ on Extension of CFD Codes to Two-Phase Flow Safety Problems, which I tried to summarize here. In alphabetical order, thanks are due to: M. Andreani (PSI, Switzerland), H. Anglart (KTH, Sweden), A. Dehbi (PSI, Switzerland), M. Heitsch (GRS, Germany), F. Kasahara (JNES, Japan), E. Komen (NRG, Netherlands), E. Laurien (IKE, Germany), D. Lucas (FZD, Germany), J. Mahaffy (Penn State Univ, USA), F. Moretti (Pisa Univ., Italy), T. Morii (JNES, Japan), P. Mühlbauer (UJV, Czech Rep.), J. Royen (OECD), M. Scheuerer (GRS, Germany), B.L. Smith (PSI, Switzerland), C.H. Song (KAERI, Korea), T. Watanabe (JAEA, Japan), G. Zigh (NRC, USA)

\section{GLOSSARY \\ BPG Best Practice Guidelines \\ BWR Boiling Water Reactor \\ CFD Computational Fluid Dynamics \\ CHF Critical Heat Flux \\ CSNI Committee on the Safety of Nuclear Installations \\ DNB Departure from Nucleate Boiling \\ DNS Direct Numerical Simulation \\ ECC Emergency Core-Cooling \\ ECCS Emergency Core-Cooling System \\ ILIS Identification of the Local Interfacial Structure \\ ITM Interface Tracking Method \\ LBLOCA Large-Break Loss Of Coolant Accident \\ LES Large Eddy Simulation \\ LOCA Loss Of Coolant Accident \\ NEA Nuclear Energy Agency \\ NRS Nuclear Reactor Safety \\ OECD Organisation for Economic Cooperation and Development \\ PIRT Process Identification and Ranking Table \\ PTS Pressurised Thermal Shock \\ PWR Pressurised Water Reactor \\ RANS Reynolds-Averaged Navier-Stokes \\ SET Separate Effect Test \\ URANS Unsteady Reynolds-Averaged Navier-Stokes \\ VLES Very Large Eddy Simulation \\ VOF Volume-Of-Fluid \\ WGAMA Working Group on the Analysis and Management of Accidents}

\section{REFERENCES}

[ 1] Mahaffy, J. (ed.) "Best Practice Guidelines for the Use of CFD in Nuclear Reactor Safety Applications", OECD, Nuclear Energy Agency, Technical Report, CSNI/R(2007)5, April 2007

[2] Smith, B.L. (ed.), “Assessment of Computational Fluid
Dynamics (CFD) for Nuclear Reactor Safety Problems", OECD Nuclear Energy Agency, Technical Report, NEA/ CSNI/R(2007)13, Jan. 2008.

[ 3 ] D. Bestion, H. Anglart, B.L. Smith, J. Royen, M. Andreani, J.H. Mahaffy, F. Kasahara, E. Komen, P. Mühlbauer, T. Morii, M. Scheuerer, E. Laurien, T. Watanabe, A. Dehbi, Extension of CFD Codes to Two-Phase Flow Safety Problems, NEA/SEN/SIN/AMA(2006)2

[ 4 ] D. Bestion, H. Anglart, J. Mahaffy, D. Lucas, C.H. Song, M. Scheuerer, G. Zigh, M. Andreani, F. Kasahara, M. Heitsch, E. Komen, F. Moretti, T. Morii, P. Mühlbauer, B.L. Smith, T. Watanabe, Extension of CFD Codes to Two-Phase Flow Safety Problems, NEA-CSNI-R(2010)2

[ 5 ] D. Bestion, H. Anglart, D. Caraghiaur, P. Péturaud, B. Smith, M. Andreani, B. Niceno, E. Krepper, D. Lucas, F. Moretti, M. C. Galassi, J. Macek, L. Vyskocil, B. Koncar, and G. Hazi, Review of Available Data for Validation of Nuresim Two-Phase CFD Software Applied to CHF Investigations, Science and Technology of Nuclear Installations, Volume 2009 (2009), Article ID 214512

[6] D. Caraghiaur and H. Anglart, Measurements and CFD predictions of velocity, turbulence intensity and pressure development in BWR fuel rod assembly with spacers, NURETH-12, Sheraton Station Square, Pittsburgh, Pennsylvania USA, 30 Septembre-4 October 2007

[ 7 ] D. Caraghiaur and H. Anglart, Annular flow deposition model with obstacle effect, 6th Int. Conf. on multiphase Flow ICMF, 2007, Leipzig,, Germany, July 9-13

[ 8 ] D. Caraghiaur and H. Anglart, Lagrangian particle tracking as a tool for deposition modelling in annular flow, ICONE17, july 12-17, 2009, Brussels, Belgium

[9] C. Chauliac, J.M. Aragonés, D. Bestion, D.G. Cacuci, N. Crouzet , F.P. Weiss, M. A. Zimmermann , NURESIM A European simulation platform for nuclear reactor safety: multi-scale and multi-physics calculations, sensitivity and uncertainty analysis, FISA 2009, Praha, Czech Rep., June 22-24, 2009

[10] D. Lucas, D. Bestion, E. Bodèle, P. Coste, M. Scheuerer, F. D'Auria, D. Mazzini, B. Smith, I. Tiselj, A. Martin, D. Lakehal, J.-M. Seynhaeve, R. Kyrki-Rajamäki, M. Ilvonen, and J. Macek, An Overview of the Pressurized Thermal Shock Issue in the Context of the NURESIM Project, Science and Technology of Nuclear Installations, Volume 2009 (2009), Article ID 583259

[11] M. Boucker, A. Guelfi, S. Mimouni, P. Péturaud, D. Bestion, E. Hervieu, Towards the prediction of local thermalhydraulics in real PWR core conditions using NEPTUNE_CFD software, Workshop on Modeling and Measurements of Two-Phase Flows and Heat Transfer in Nuclear Fuel Assemblies, KTH, Stockholm, Sweden - 1011 October 2006

[12] Yao W., Morel C., 2004, Volumetric interfacial area prediction in upward bubbly two-phase flow, Int. J. Heat \& Mass Transfer, Vol. 47, pp. 307-328.

[13] Morel, C., Wei Yao, Bestion, D., Three Dimensional modelling of boiling flow, The 10th International Topical Meeting on Nuclear Reactor Thermal Hydraulics (NURETH-10), Seoul, Korea, October 5-9, 2003

[14] Morel, C., Mimouni, S., Lavieville, M. and Boucker, M., 2005, R113 Boling Bubbly Flow in an annular Geometry simulated with the NEPTUNE code, 11 th Int. Topical 
Meeting on Nuclear Reactor Thermal-Hydraulics (NURETH-11), Avignon, France, October 2-6.

[15] C. Morel and J. M. Laviéville, Modeling of Multisize Bubbly Flow and Application to the Simulation of Boiling Flows with the Neptune CFD Code, Science and Technology of Nuclear Installations, Volume 2009, Article ID 953527

[16] D. Lucas, E. Krepper, H.M. Prasser, Modelling of the evolution of large bubbly flow along a vertical pipe, 2007, Nuclear Technology 158, 291-303

[17] S. Mimouni, F. Archambeau, M. Boucker, J. Laviéville, CFD Modelling of subcooled boiling in the NEPTUNE_ CFD code and application to fuel assembly analysis, XCFD4NRS, GRENOBLE, FRANCE, Sept10-12, 2008

[18] S. Mimouni, F. Archambeau, M. Boucker, J. Laviéville, and C. Morel, A Second-Order Turbulence Model Based on a Reynolds Stress Approach for Two-Phase FlowPart I: Adiabatic Cases, Science and Technology of Nuclear Installations, Volume 2009 (2009), Article ID 792395

[19] B. Ničeno, M. Boucker, and B. L. Smith, Euler-Euler Large Eddy Simulation of a Square Cross-Sectional Bubble Column Using the Neptune_CFD Code, Science and Technology of Nuclear Installations, Volume 2009 (2009), Article ID 410272

[20] Koncar, B., Use of two-phase wall function for simulation of boiling flow, companion paper proposed at NURETH 12, Pittsburgh, Pennsylvania, U.S.A. September 30-October 4, 2007

[21] B. Koncar, E. Krepper, CFD simulation of convective flow boiling of refrigerant in a vertical annulus, Nucl. Eng. Des. Volume 238, Issue 3, 2008, pp. 693-706

[22] C. Morel and J. M. Laviéville, Modeling of Multisize Bubbly Flow and Application to the Simulation of Boiling Flows with the Neptune CFD Code, Science and Technology of Nuclear Installations, Volume 2009, Article ID 953527,

[23] Krepper, E.; Frank, T.; Lucas, D.; Prasser, H.-M.; Zwart, Philip J., 2007, Inhomogeneous MUSIG model - a population balance approach for polydispersed bubbly flows, The 12th International Topical Meeting on Nuclear Reactor Thermal Hydraulics (NURETH-12), 30.09.-04.10. 2007, Pittsburgh, USA

[24] E. Krepper, P. Ruyer, M. Beyer, D. Lucas, H.-M. Prasser, and N. Seiler, CFD Simulation of Polydispersed Bubbly Two-Phase Flow around an Obstacle, Science and Technology of Nuclear Installations, Volume 2009 (2009), Article ID 320738

[25] J. Macek, L. Vyskocil, Simulation of Critical Heat Flux Experiments in NEPTUNE_CFD Code XCFD4NRS, Grenoble, France, 10 - 12 September 2008

[26] D. Lucas, D. Bestion, E. Bodèle, P. Coste, M. Scheuerer, F. D'Auria, D. Mazzini, B. Smith, I. Tiselj, A. Martin, D. Lakehal, J.-M. Seynhaeve, R. Kyrki-Rajamäki, M. Ilvonen, and J. Macek, An Overview of the Pressurized Thermal Shock Issue in the Context of the NURESIM Project, Science and Technology of Nuclear Installations, Volume 2009 (2009), Article ID 583259

[27] Y. Bartosiewicz, J.-M Laviéville and J.-M Seynhaeve, "A first Assessment of the NEPTUNE_CFD code: Instabilities in a Stratified Flow, Comparison between the VOF Method and a Two-Field Approach", International Journal of Heat and Fluid Flow, vol. 29, pp. 460-478, 2008.

[28] Yao, W., Coste, P., Bestion, D., Boucker, M., 2003, TwoPhase Pressurised Thermal Shock investigations using a 3D 2-Fluid Modelling of stratified Flow with Condensation, The 10th International Topical Meeting on Nuclear Reactor Thermal Hydraulics (NURETH-10), Seoul, Korea, October 5-9, 2003

[29] P. Coste, J. Pouvreau, J. Laviéville, M. Boucker, "Status of a two-phase CFD approach to the PTS issue". XCFD4NRS, Grenoble, France, 10 - 12 September 2008a

[30] D. Lakehal: LEIS for the Prediction of Turbulent Multifluid Flows Applied to Thermal Hydraulics Applications. XCFD4NRS, Grenoble, Sep. 10-12, 2008

[31] Lakehal D., Fulgosi M., Banerjee S., Yadigaroglu G.,: Turbulence and heat transfer in condensing vapor-liquid flow, Phys. Fluids, 20, 065101, 2008.

[32] Y. Bartosiewicz, J.M. Seynhaeve, C. Vallée, T. Höhne, J.M. Laviéville, Modelling free surface flows relevant to a PTS scenario: comparison between experimental data and three RANS based CFD-codes, XCFD4NRS, Experiments and CFD Code Applications to Nuclear Reactor Safety OECD/NEA \& IAEA, Grenoble, France, 10 - 12 September 2008

[33] M.C. Galassi, C. Morel, D. Bestion, J. Pouvreau, F. D’Auria, Validation of NEPTUNE CFD Module with Data of a Plunging Water Jet Entering a Free Surface, NURETH-12, Pittsburgh, Pennsylvania USA, 30 September-4 October 2007

[34] M. Scheuerer, M.C. Galassi, P. Coste, F. D'Auria, Numerical simulation of free surface flow with heat and mass transfer, NURETH-12, Pittsburgh, Pennsylvania USA, 30 September-4 October 2007

[35] L. Štrubelj, I. Tiselj, Numerical simulation of vapour condensation on highly subcooled liquid surface, XCFD4NRS, Experiments and CFD Code Applications to Nuclear Reactor Safety OECD/NEA \& IAEA, Grenoble, France, 10 - 12 September 2008

[36] C.-H. Song et al., "Development of the PIRT for Thermal Mixing Phenomena in the APR1400 IRWST", Proc. 5th Korea-Japan Symposium on Nuclear Thermal-Hydraulics and Safety (NTHAS5), Jeju, Korea, Nov. 26-29 (2006).

[37] C.-H. Song et al., "Characterization of Direct Contact Condensation of Steam Jets Discharging into a Subcooled Water", Proc. IAEA Technical Committee Mtg., PSI, Villigen, Sept. 14 (1998)

[38] B.L. Smith, M. Milelli, S. Shepel \& D. Lakehal, "A Generic Study of Phenomena Affecting Two-Phase Mixing in BWR Suppression Pools during Passive Removal", Proc. NURETH-10, Seoul, Korea, Oct. 5-9 (2003).

[39] Y.J. Choo \& C.-H. Song, "PIV Measurements of Turbulent Jet and Pool Mixing Produced by a Steam Jet in a Subcooled Water Pool", Proc. XCFD4NRS workshop, Grenoble, Sept. 10 (2008)

[40] H.S. Kang \& C.-H. Song, "CFD Analysis of a Thermal Mixing in a Subcooled Water Pool Under a High Steam Mass Flux", Proc. CFD4NRS Workshop, Garching, Germany, Sept. 5-7 (2006a).

[41] H.S. Kang \& C.-H. Song, "CFD Analysis of a Turbulent Jet Behavior Induced by a Steam Jet Discharged through a Single Hole in a Subcooled Water Pool", Proc. XCFD4NRS workshop, Grenoble, Sept. 10 (2008) 